\title{
Flexible PDMS-based triboelectric nanogenerator for instantaneous force sensing and human joint movement monitoring
}

\author{
Junbin $\mathrm{Yu}^{1 \dagger}$, Xiaojuan $\mathrm{Hou}^{1 \dagger}$, Min $\mathrm{Cui}^{1}$, Shuzheng Shi ${ }^{1,2}$, Jian $\mathrm{He}^{1}$, Yawei Sun ${ }^{3}$, Chao Wang and \\ Xiujian Chou ${ }^{1 *}$
}

\begin{abstract}
Flexible wearable sensors with excellent electric response and self-powered capability have become an appealing hotspot for personal healthcare and human-machine interfaces. Here, based on triboelectric nanogenerator (TENG), a flexible self-powered tactile sensor composed of micro-frustum-arrays-structured polydimethylsiloxane (PDMS) film/copper (Cu) electrodes, and poly(vinylidenefluoride-trifluoroethylene) (P(VDF-TrFE)) nanofibers has been demonstrated. The TENG-based self-powered tactile sensor can generate electrical signals through the contact-separation process of two triboelectric layers under external mechanical stimuli. Due to the uniform and controllable micro-frustum-arrays structure fabricated by micro-electro-mechanical system (MEMS) process and the $P$ (VDF-TrFE) nanofibers fabricated by electrostatic spinning, the flexible PDMS-based sensor presents high sensitivity of $2.97 \mathrm{~V} \mathrm{kPa}^{-1}$, stability of 40,000 cycles (no significant decay), response time of $60 \mathrm{~ms}$ at $1 \mathrm{~Hz}$, low detection pressure of a water drop ( 4 Pa, $35 \mathrm{mg}$ ) and good linearity of 0.99231 in low pressure region. Since the PDMS film presents ultra-flexibility and excellent-biocompatibility, the sensor can be comfortably attached on human body. Furthermore, the tactile sensor can recognize various types of human body movements by the corresponding electrical signals. Therefore, the as-prepared TENGs are potential on the prospects of gesture detection, health assessment, human-machine interfaces and so on.
\end{abstract}

Keywords: self-powered, triboelectric nanogenerator, wearable sensor, MEMS process, personal healthcare

\section{INTRODUCTION}

Monitoring various human body motions has been considered as an effective way for gesture detection and health assessment. Recently, flexible tactile sensors are drawing significant attention due to the unique capability to mimic human skin perception of sensing subtle pressure changes, which are potential in biomimetic prostheses [1], mobile medical diagnostics [2,3], non-invasive health care [4-6] and human-machine interactions [7-9]. Conventional healthcare sensing devices including highspeed camera, photo-electric devices [10] and siliconbased sensors [11] have been employed for monitoring body motion; however the applications are still limited because of their poor flexibility and wearability. To avoid discomfort for the user, the flexible and stretchable substrates are crucial factors to be considered. Accordingly, polydimethylsiloxane (PDMS), which can be discretionarily stretched, twisted, compressed and deformed, has become a common substrate material in chemistry, physics, materials science, mechanical engineering and microelectronics [12-14]. Benefiting from ultra-flexibility and excellent biocompatibility, PDMS films are the most popular flexible substrate to integrate various sensitive materials which have promoted the rapid development of flexible tactile sensor to monitor human body movement $[15,16]$. Various micro-structured PDMS films (leavepatterned [17], silk-patterned [18], micro pyramid [19] and so on) are fabricated through all kinds of molds as the key element of the flexible sensor, which could store

\footnotetext{
${ }^{1}$ Science and Technology on Electronic Test and Measurement Laboratory, School of Instrument and Electronics, North University of China, Taiyuan 030051, China

${ }^{2}$ School of Mechanical Engineering, Hebei University of Architecture, Zhangjiakou 075000, China

${ }^{3}$ North Information Control Research Academy Group Co., Ltd, Nanjing 211100, China

These authors contributed equally to this paper.

* Corresponding author (email: chouxiujian@nuc.edu.cn)
} 
and release the external energy with huge elastical deformation due to low elastic modulus and micro-structure [18]. Furthermore, the PDMS film with high-density micro-structure can effectively solve the trouble of viscoelastic behaviour. Hence, the preparation of well-controllable and regular micro-patterned PDMS film has been extensively studied $[12,20]$.

Recently, a series of new flexible tactile sensors based on piezoelectric nanogenerators (PENG) $[21,22]$ and triboelectric nanogenerators (TENG) $[20,23,24]$ have been widely studied due to their exciting potential as mimic human skin with no need for traditional power supplies. PENG-based self-powered tactile sensors demonstrate ultrafast response, high sensitivity and great potential applications in flexible tactile device. Nevertheless, the necessary polarization process of piezoelectric materials severely limits their application [25]. By contrast, TENGs transform mechanical energy to electricity by coupling triboelectrification and electrostatic induction, which can directly generate electrical signals without any pre-treatment and present potential in low-frequency mechanical stimuli harvesting and self-powered sensing [26,27]. The TENG is usually composed of two friction layers with corresponding electrodes, and has been widely studied due to the simple fabrication, low cost and environmental friendliness [28]. When an external force is applied, the two friction layers can simultaneously contact and rub each other with suitable structure design. In order to increase the sensitivity of TENG-based flexible tactile sensor, the relatively rigid film materials were currently chosen as active layers to transfer external mechanical energy [29]. And it is also necessary to increase the specific surface area of the friction layers and select the materials with high capacity to donate/gain electrons as triboelectric pair, such as polyethylene terephthalate (PET), Kapton and metal film with micro/nano-structure [29]. However, most of the selected materials cannot fully contact with irregular skin and cause uncomfortableness for people. PDMS not only presents excellent flexibility, but also shows strong ability to gain electrons as a negative friction material in the friction series. Hence, the integration of flexible micro-structured PDMS into TENG for self-powered sensor with high sensitivity brings new vitality and more possibilities for next-generation tactile sensor.

In this study, through micro-electro-mechanical system (MEMS) process, we fabricated a flexible self-powered triboelectric tactile sensor for sensing instantaneous force and monitoring human joint movement, which is composed of a spacer layer, poly(vinylidenefluoride-tri- fluoroethylene) (P(VDF-TrFE)) nanofibers and $\mathrm{Cu} /$ PDMS films with uniform and controllable micro-frustum-arrays structure $(60 \mu \mathrm{m} \times 60 \mu \mathrm{m} \times 39 \mu \mathrm{m}$ at intervals of $60 \mu \mathrm{m})$ (mf-Cu/PDMS). Benefiting from the enhanced sensitivity caused by the triboelectric pair of $\mathrm{mf}-\mathrm{Cu} /$ PDMS, the flexible TENG shows good sensitivity $\left(2.97 \mathrm{~V} \mathrm{kPa}^{-1}\right)$ and thus detects instantaneous and tiny force applied by a water drop ( 4 Pa, $35 \mathrm{mg})$. P(VDFTrFE) nanofibers are deposited on the mf-PDMS by electrospinning, which can effectively reduce the adhesion of the two friction layers and then increase the sensor linearity. Importantly, through the loading/unloading test, the sensors present well durability (more than 40,000 pressure cycles without significant decay) and fast response time $(60 \mathrm{~ms}$ at $1 \mathrm{~Hz})$. For human body motion detection, sensors were easily and fully attached on irregular skin to distinguish the types of gesture by the differences of generated electric signals. Therefore, the asprepared flexible tactile sensors are able to sense instantaneous force and detect human joint motion movement, which may be applied in personal healthcare and human-machine interfaces.

\section{EXPERIMENTAL SECTION}

\section{Materials}

Tetramethylammonium hydroxide (TMAH, the mass ratio of TMAH to $\mathrm{H}_{2} \mathrm{O}$ is $10 \%$ ), buffered oxide etch (BOE, $\mathrm{NH}_{4} \mathrm{~F}: \mathrm{H}_{2} \mathrm{O}=5: 1$ ), hydrochloric acid solution (50 wt\% in $\mathrm{H}_{2} \mathrm{O}$ ), acetone (AR, 99.5\%), $\mathrm{N}, \mathrm{N}$-dimethylformamide (DMF, AR, 99.9\%), PDMS and curing agent (Sylgard 184 purchases from Dow Corning), P(VDF-TrFE) (80/20 wt\% of PVDF/TrFE), the Si wafer (100, 4-inch with $300 \mathrm{~nm}$ $\mathrm{SiO}_{2}$ layer) and RZJ304 photoresist were provided by RDMICRO.

\section{Fabrication of the silicon template}

The micropattern on the pre-engineered mask $(60 \mu \mathrm{m} \times$ $60 \mu \mathrm{m})$ was transferred to the $\mathrm{Si}$ wafer by photolithography technology. Then the exposed $\mathrm{SiO}_{2}$ layer was etched by immersing the Si wafer in the BOE solution at $80^{\circ} \mathrm{C}$ for $5 \mathrm{~min}$, followed by immersing in TMAH solution at $80^{\circ} \mathrm{C}$ for $30 \mathrm{~min}$ to obtain the concave microfrustum-arrays structure $(60 \mu \mathrm{m} \times 60 \mu \mathrm{m} \times 39 \mu \mathrm{m}$ with interval of $60 \mu \mathrm{m}$ ).

\section{Fabrication of micro-frustum-arrays structure PDMS (mf-} PDMS) films

The mixture of PDMS and curing agent (10:1) was stirred for $20 \mathrm{~min}$ and degassed in vacuum for $20 \mathrm{~min}$ to remove 
the bubbles. The $100 \mathrm{~nm} \mathrm{Al} \mathrm{film} \mathrm{was} \mathrm{sputtered} \mathrm{on} \mathrm{the} \mathrm{Si}$ mold, and then the PDMS mixture was spin-coated onto the Si template and solidified at $80^{\circ} \mathrm{C}$ for $30 \mathrm{~min}$. The $\mathrm{Si}$ template (with PDMS film) was immersed in hydrochloric acid solution and ultrasonicated for $30 \mathrm{~min}$ to get an mf-PDMS film.

\section{Fabrication of $\mathrm{P}(\mathrm{VDF}-\mathrm{TrFE})$ nanofibers}

The $\mathrm{P}(\mathrm{VDF}-\mathrm{TrFE})$ polymer $(1.8 \mathrm{~g}), \mathrm{DMF}(4 \mathrm{~mL})$ and acetone $(6 \mathrm{~mL})$ were added into a beaker, and stirred at room temperature for $6 \mathrm{~h}$. The $\mathrm{P}(\mathrm{VDF}-\mathrm{TrFE})$ nanofibers were fabricated by electrostatic spinning process with an applied voltage of $10 \mathrm{kV}$ and $-1.5 \mathrm{kV}$, collector-nozzle distance of $20 \mathrm{~cm}$, and feeding speed of $0.15 \mathrm{~mm} \mathrm{~min}^{-1}$ under the ambient environment.

\section{Fabrication of TENG self-powered tactile sensor}

The $\mathrm{P}(\mathrm{VDF}-\mathrm{TrFE})$ nanofibers were directly deposited on the $\mathrm{mf}$-PDMS film (as the top friction layer, $15 \mathrm{~mm} \times$ $15 \mathrm{~mm} \times 0.13 \mathrm{~mm}$ ) by electrostatic spinning process. The $200 \mathrm{~nm} \mathrm{Cu}$ was sputtered onto the back of the top friction layer as the top electrode. Then $200 \mathrm{~nm} \mathrm{Cu}$ was sputtered onto another $\mathrm{mf}$-PDMS $(20 \mathrm{~mm} \times 18 \mathrm{~mm} \times 0.13 \mathrm{~mm})$ film surface to obtain $\mathrm{mf}-\mathrm{Cu}$ as bottom triboelectric layer and simultaneously bottom electrode. To keep the flexibility of the sensor, the conductive fabric was connected with two $\mathrm{Cu}$ electrode layers, respectively. A spacer layer (50 $\mu \mathrm{m})$ with hollow structure was placed between the two mf-PDMS films to form a cavity structure. The top mfPDMS film, spacer layer and bottom mf-PDMS film were stacked in order and then covered by the pure PDMS film.

\section{Characterization}

The sample surface morphology was characterized by scanning electron microscopy (SEM, ZEISS EVO18, Carl Zeiss Jena, Germany). X-ray diffraction (XRD) analysis was carried out using the X-ray diffractometer DX-2700X (Dandong fangyuan instrument co. LTD, China). The output voltage and current were measured with 2611B Source Meter test system (KEITHLET).

\section{RESULTS AND DISCUSSION}

Fig. 1a shows the fabrication process of the TENG-based flexible sensor. The Si template with uniform microfrustum-arrays structure was prepared by MEMS manufacturing technology. Al was deposited on the Si template by magnetron sputtering and then PDMS was spin-coated
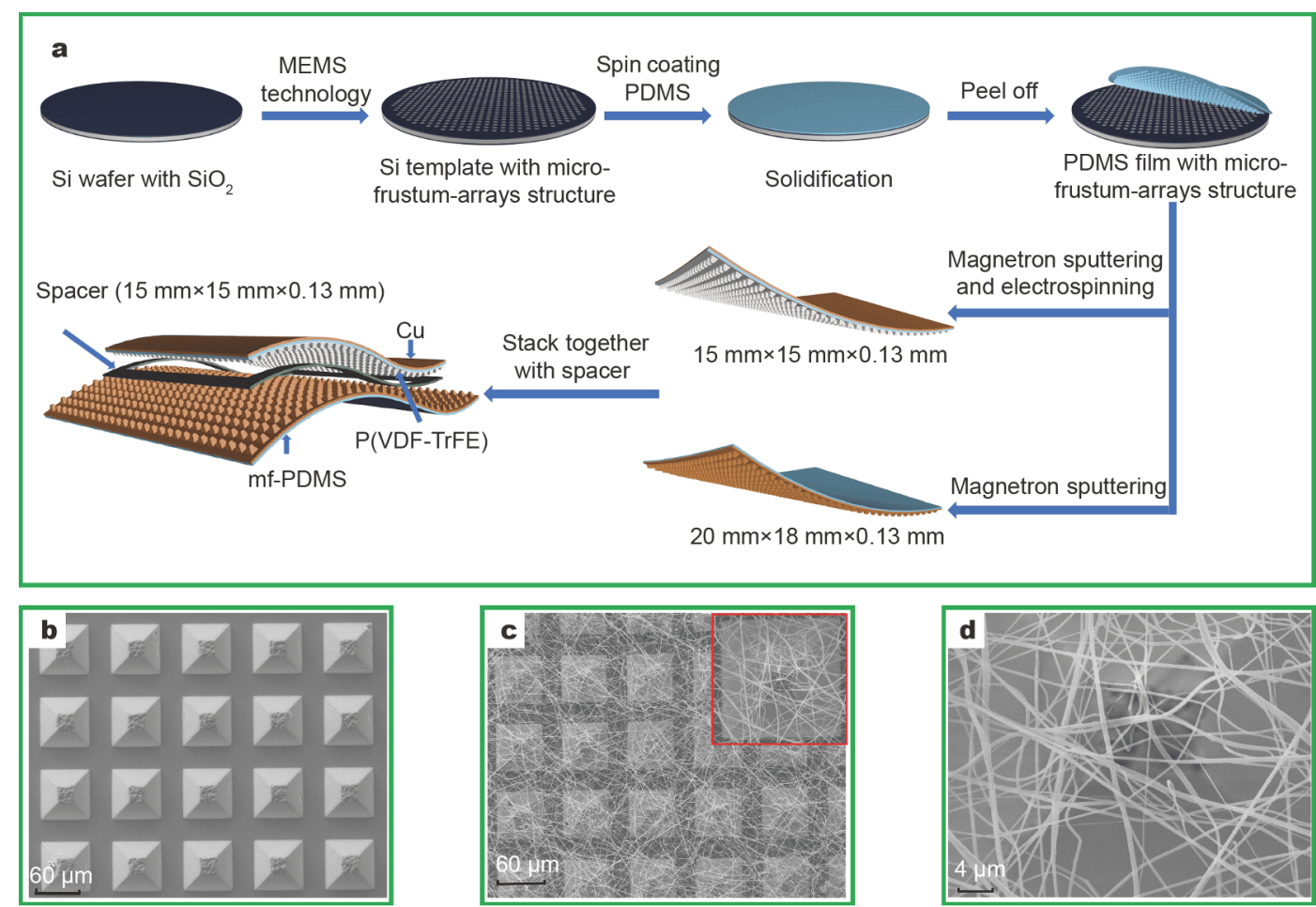

Figure 1 (a) Schematic illustration of the fabrication process for the flexible mf-PDMS based TENG sensor; (b) SEM image of the mf-PDMS film; (c, d) SEM images of the mf-PDMS film with P(VDF-TrFE) nanofibers. 
onto the Si mold and solidified at $80^{\circ} \mathrm{C}$ for $30 \mathrm{~min}$. The $\mathrm{Si}$ mold with PDMS was immersed in hydrochloric acid solution and ultrasonicated for $30 \mathrm{~min}$ to easily peel off the entire mf-PDMS film $(60 \mu \mathrm{m} \times 60 \mu \mathrm{m} \times 39 \mu \mathrm{m}$ at intervals of $60 \mu \mathrm{m}$ ) without any adhesion to the Si mold. Fig. $1 \mathrm{~b}$ shows the SEM image of mf-PDMS film. Cu film was deposited on the back of mf-PDMS $(15 \mathrm{~mm} \times 15 \mathrm{~mm}$ $\times 0.13 \mathrm{~mm})$ as electrode and the $\mathrm{P}(\mathrm{VDF}-\mathrm{TrFE})$ nanofibers were directly deposited on the front of its surface for $5 \mathrm{~min}$, which aim to reduce the adhesion between two triboelectric layers and increase the linearity of sensor. The mf-PDMS thin film $(15 \mathrm{~mm} \times 15 \mathrm{~mm} \times 0.13 \mathrm{~mm})$ served as one triboelectric layer. Fig. 1c, $d$ show the SEM images of mf-PDMS film with P(VDF-TrFE) nanofibers. $\mathrm{Cu}$ film $(200 \mathrm{~nm})$ was deposited on another mf-PDMS $(20 \mathrm{~mm} \times 18 \mathrm{~mm} \times 0.13 \mathrm{~mm}$ ) film surface to obtain $\mathrm{mf}-\mathrm{Cu}$ as another triboelectric layer and simultaneously electrode. The spacer layer $(50 \mu \mathrm{m})$ with hollow structure was placed between the two mf-PDMS films to form a cavity structure, leading to the perfect implementation of contact-separation process.

Here, PDMS was chosen due to its outstanding ability of gaining electrons and excellent flexibility. Employing PDMS as the main body and MEMS technology as the preparation process, the sensor presents the characteristics of ultra-thin $(0.56 \mathrm{~mm})$, small size (the same size as 50 cents coin), excellent flexibility (bent nearly $180^{\circ}$ ) and super-light $(0.155$ g) (Fig. 2a-c and Fig. S1a). These characteristics make it possible for the TENG-based sensor to be easily attached onto human skin or soft fabrics for gesture detection, health assessment and so on.

Fig. 2d illustrates the operating principle of TENGbased sensor. At the original state, two triboelectric layers separate from each other and the sensor shows electrically neutral state without charges. When mf-PDMS and $\mathrm{mf}-$ $\mathrm{Cu}$ come into contact and rub with each other by external force, the opposite surface charges with equal amounts are distributed on the contact surface of two triboelectric layers due to the triboelectric effect. According to the triboelectric series, electrons transfer from $\mathrm{mf}-\mathrm{Cu}$ to $\mathrm{mf}$ PDMS, which causes the negative charges to accumulate on mf-PDMS surface and the positive charges to accumulate on $\mathrm{mf}-\mathrm{Cu}$ surface. When the external force is released, the electrons will flow from the $\mathrm{Cu}$ electrode to the $\mathrm{mf}-\mathrm{Cu}$ electrode through external load to keep the electrostatic neutral state. Finally, the upper PDMS film is completely restored to the initial state and the sensor shows the electrostatic neutral state. When the external

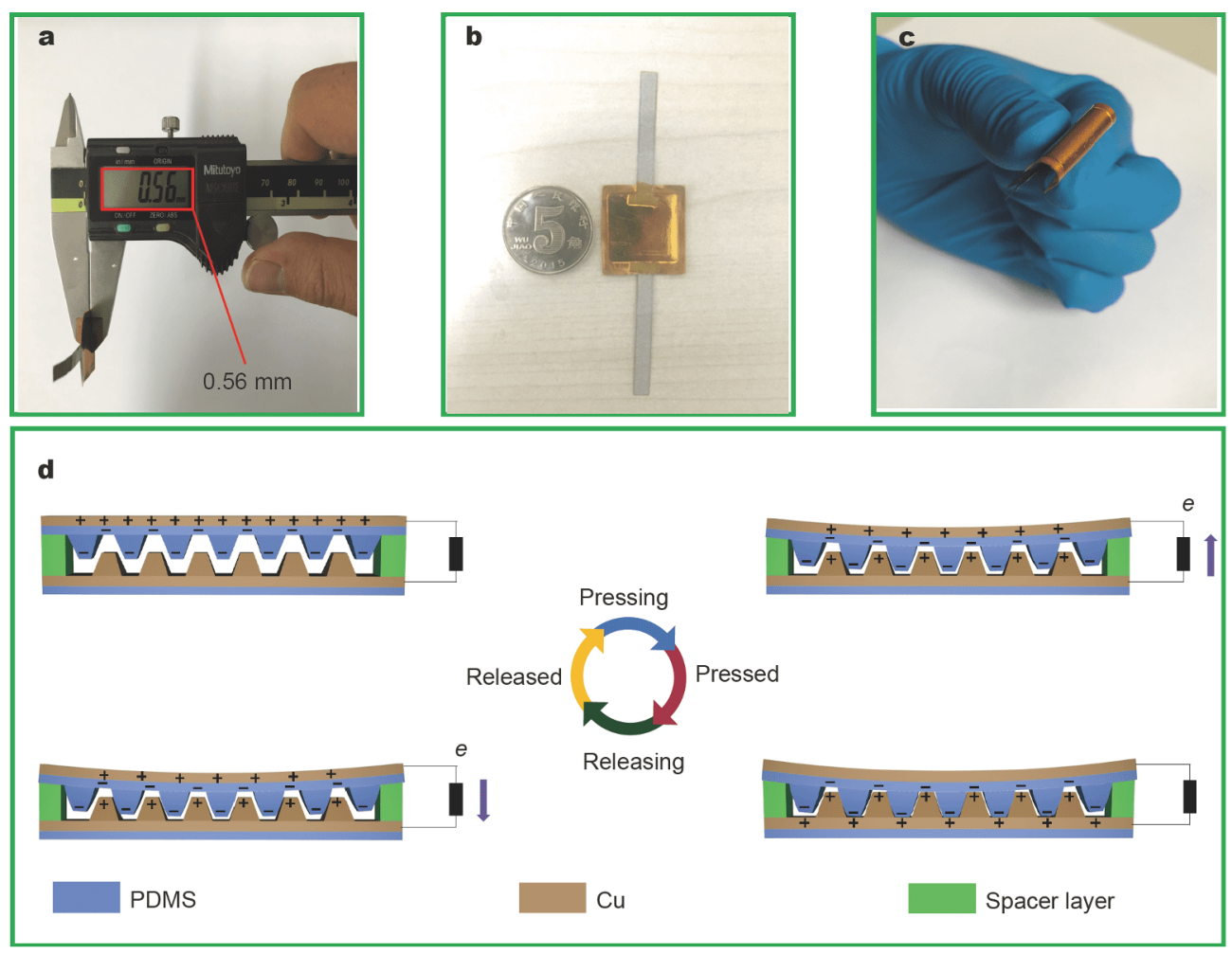

Figure $2(a-c)$ Photographs of TENG show the thickness of $0.56 \mathrm{~mm}$, small size and excellent flexibility; (d) the electrical signals generation mechanism of TENG. 
force is applied to the TENG sensor again, the electrostatic equilibrium is disrupted and electrons will flow from $\mathrm{mf}-\mathrm{Cu}$ electrode to $\mathrm{Cu}$ electrode through the external load. Hence, under continuous compressing and releasing, the sensor can generate alternating electrical signals, which match with the external force. In the opencircuit condition, the open-circuit voltage $V_{\mathrm{OC}}$ can be expressed as [30]

$V_{\mathrm{OC}}=\sigma x(t) / \varepsilon_{\mathrm{air}}$,

where $\varepsilon_{\text {air }}$ is the relative dielectric constant of the cavity, $\sigma$ is the triboelectric charge density and $x(t)$ is the distance of the two triboelectric layers. According to the Hooker's Law, the deformation of PDMS film is proportional to the applied external force [20]. Thus, the $V_{\mathrm{OC}}$ can also be denoted as

$\left(V_{\mathrm{OC}, 0}-V_{\mathrm{OC}}\right) / V_{\mathrm{OC}}=[x(0)-x(t)] / x(0)=S P /[k x(0)]$,

where $S$ is the effective contact area of the two friction layers, $P$ is the applied pressure and $k$ is the elastic property of the material of the flexible TENG pressure sensor.
The $V_{\mathrm{OC}}$ is related to the charge density, and the charge density depends on the choice of friction materials and the specific surface area of friction layer. $\mathrm{Cu}$ and PDMS are located at opposite ends of triboelectric series, showing excellent capacity of losing and gaining electrons. In terms of surface morphology, the previous study about TENG has investigated the output performance with different micro-structured films, and the output follows the following relationship: film $<$ line $<$ cube $<$ pyramid [31]. However, they did not study the output performance under the micro-frustum-arrays structure. Here, the home-made test system (Fig. S2a) was designed to investigate the output performance of TENG-based sensor with micro-pyramid-arrays PDMS (mp-PDMS, the SEM image was shown in Fig. S1b), mf-PDMS without $\mathrm{P}(\mathrm{VDF}-\mathrm{TrFE})$ nanofibers and mf-PDMS with $\mathrm{P}(\mathrm{VDF}-\mathrm{TrFE})$ nanofibers.

As shown in Fig. 3a, b, the results present the output under different surface patterns: mp-PDMS with P(VDFTrFE) nanofibers $<$ mf-PDMS with $\mathrm{P}(\mathrm{VDF}-\mathrm{TrFE})$ nanofibers $\approx$ mf-PDMS without $\mathrm{P}(\mathrm{VDF}-\mathrm{TrFE})$ nanofibers, and
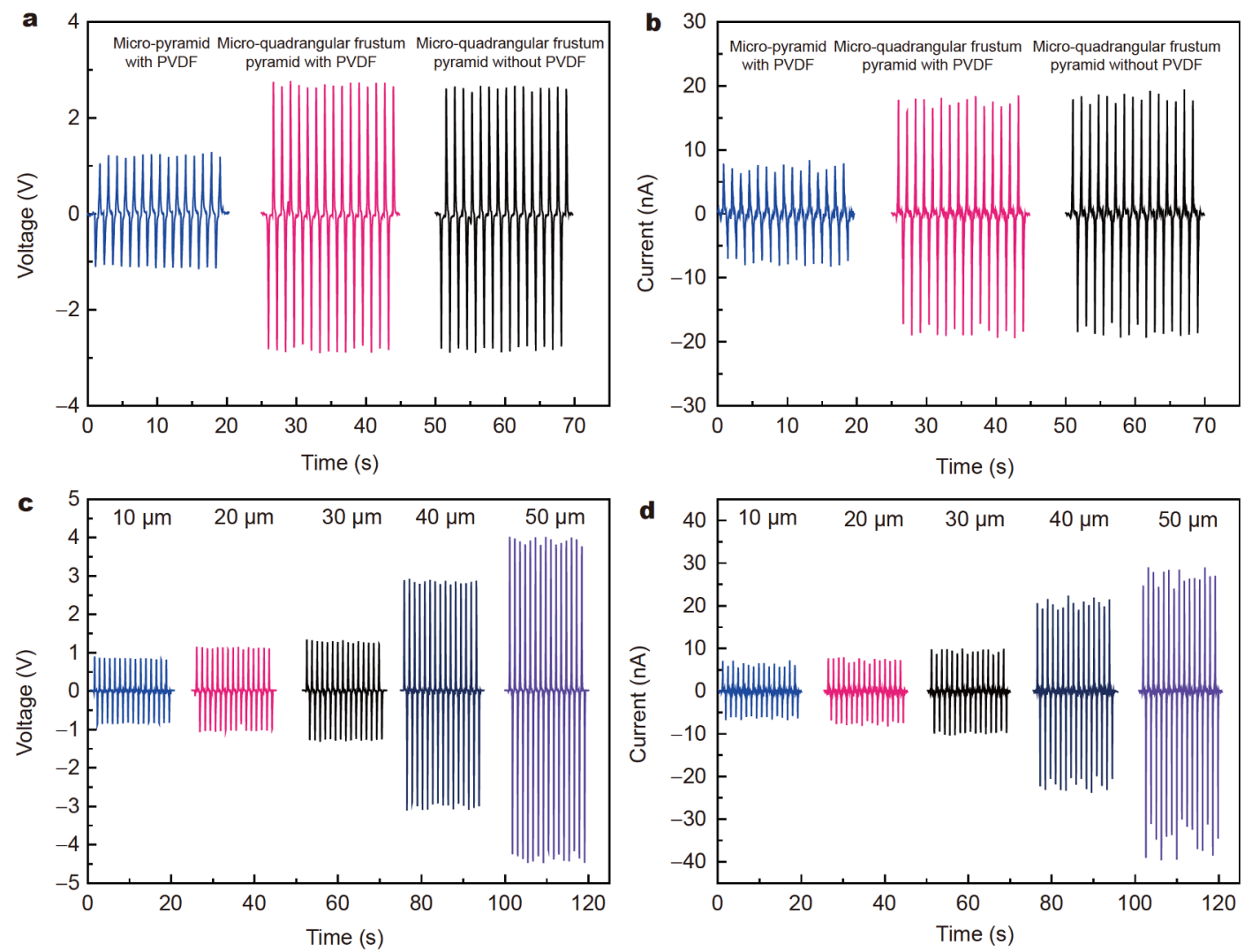

Figure $3(\mathrm{a}, \mathrm{b})$ The open-circuit voltage and short-circuit current output of the TENG with different micro structures of the triboelectric layer under applied pressure of $10 \mathrm{kPa}$. (c, d) The open-circuit voltage and short-circuit current output of the TENG with different spacer thicknesses under applied pressure of $20 \mathrm{kPa}$. 
the outputs reach up to $1.2 \mathrm{~V}, 7.6 \mathrm{nA} ; 2.6 \mathrm{~V}, 18.4 \mathrm{nA}$ and $2.7 \mathrm{~V}, 18.2 \mathrm{nA}$, respectively. The micro-frustum-arrays structure facilitates the process of triboelectrification than micro-pyramid-arrays structure. The P(VDF-TrFE) nanofibers have no obvious effect on the output performance. In other words, the micro-frustum-arrays structure helps improve the sensitivity of TENG-based sensor. In addition, it is also necessary to investigate the influence of spacer thickness on the output performance. As shown in Fig. 3c, d and Fig. S2b, c, as the spacer thickness increases, the output voltage/current shows a trend of increasing first and then decreasing. And the voltage/ current output reaches the maximum when the spacer thickness is $50 \mu \mathrm{m}$. However, the Equation (1) presents the $V_{\mathrm{OC}}$ is positively correlated with the spacer thickness. The reason for the difference is that the TENG sensor is no longer equivalent to a plane-parallel capacitor with the spacer thickness increasing [30]. Therefore, the optimal spacer thickness was selected as $50 \mu \mathrm{m}$.

Sensitivity and linearity are two important factors for sensor performance. Fig. 4a, c show the open-circuit voltage and short-circuit current of TENG with P(VDFTrFE) nanofibers at different forces from $0 \mathrm{~Pa}$ to $900 \mathrm{kPa}$. Fig. 4 b, d show the peak-to-peak voltage/current variation with different input forces and the sensor sensitivity reaches up $2.97 \mathrm{~V} \mathrm{kPa}^{-1}$ in the low-pressure region $(S=\Delta V / \Delta P)$. Moreover, the $R^{2}$ is 0.99231 and 0.93721 in the region 1 and region 2 of voltage, respectively, and 0.98665 and 0.89776 in the region 1 and region 2 of current, respectively. As shown in Fig. 3a, P(VDF-TrFE) has no significant improvement on the output of TENG. To confirm the influence of the $\mathrm{P}(\mathrm{VDF}-\mathrm{TrFE})$, we further operated the linear test of TENG without $\mathrm{P}(\mathrm{VDF}-\mathrm{TrFE})$ nanofibers at different forces from $0 \mathrm{~Pa}$ to $550 \mathrm{kPa}$ (Fig. S3a, c). As shown in Fig. S3b, d, compared with the sensor with $\mathrm{P}(\mathrm{VDF}-\mathrm{TrFE})$ nanofibers, the sensor without $\mathrm{P}(\mathrm{VDF}-\mathrm{TrFE})$ nanofibers has smaller linearity $\left(R^{2}=0.85188\right.$ of voltage and $R^{2}=0.94626$ of current). Therefore, the $\mathrm{P}(\mathrm{VDF}-\mathrm{TrFE})$ nanofibers can effectively improve the linearity of TENG sensor due to the reduced viscosity between the two friction layers. Since the $\mathrm{P}(\mathrm{VDF}-\mathrm{TrFE})$ nanofibers were directly deposited on the mf-PDMS surface and the thickness was much smaller than the PDMS thickness, the surface morphology would not change significantly and thus the spinning time would not affect the sensor output performance. The voltage and current output variation of the sensor with $\mathrm{P}(\mathrm{VDF}-\mathrm{TrFE})$ nanofibers show two linear regions. The amount of charges generated by the triboelectrification has been determined by the friction materials and the surface morphology. If we define the $L$ as the increase of effective contact area under per unit force. Then, in the low region (region 1), the $L$ shows great changes; in the high region (region 2 ), the changes of $L$ are very small as
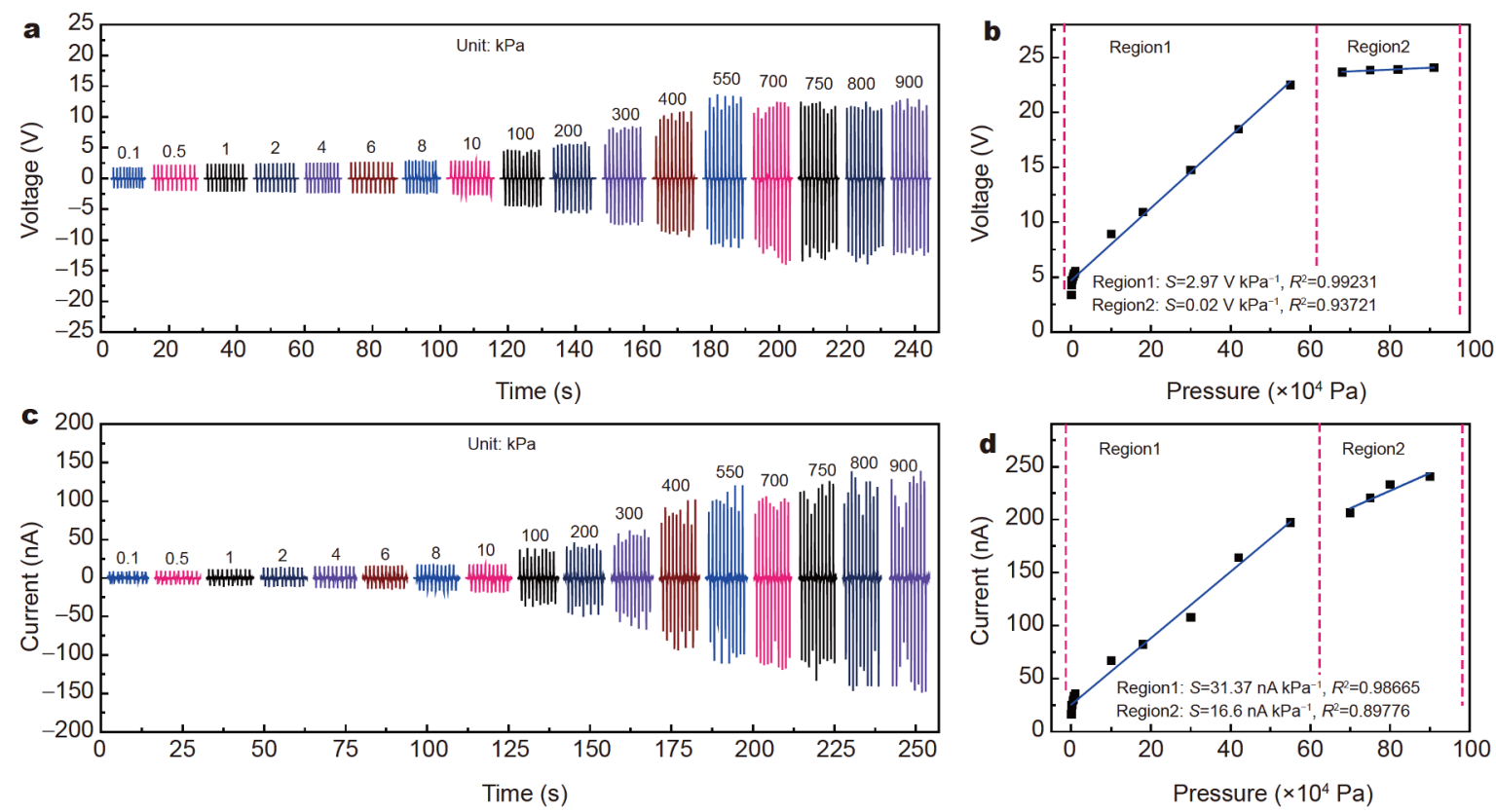

Figure 4 The output of the TENG under different pressures. (a) The open-circuit voltage; (b) the linear fitting analysis of peak-to-peak open-circuit voltage; (c) the short-circuit current; (d) the linear fitting analysis of peak-to-peak short-circuit current. 
the distance of two friction layers tends to be zero. Hence, there is no more effective friction to generate new charges and the $V_{\mathrm{OC}} / I_{\mathrm{SC}}$ tends to saturate in the region 2. Moreover, the PDMS film also has two linear regions in the stress-strain responses (easier to deform under low force region).

The TENG device as self-powered tactile sensor can directly generate voltage or current output through deformation of two triboelectric layers [32,33]. With such high sensitivity, our flexible TENG can detect the external pressure as small as the weight of a drop of water $(\sim 4 \mathrm{~Pa}$ and $35 \mathrm{mg}$, Fig. 5a, b and Movie S1). As shown in the enlarged graph of Fig. 5b, the obtained curves have similar shapes and characteristics. In Fig. 5c, the sensor was applied with force by the empty capsule $(96 \mathrm{mg}$ ) for the loading and unloading process and the response voltage signal curves are shown in Fig. 5d. These results prove that the as-prepared sensor presents high sensitivity and great repeatability. The signal of the sensor represents a sharp peak instead of a smooth state curve and demonstrates fast response and no hysteresis during fast loading and unloading process. Fig. S4 presents the response time

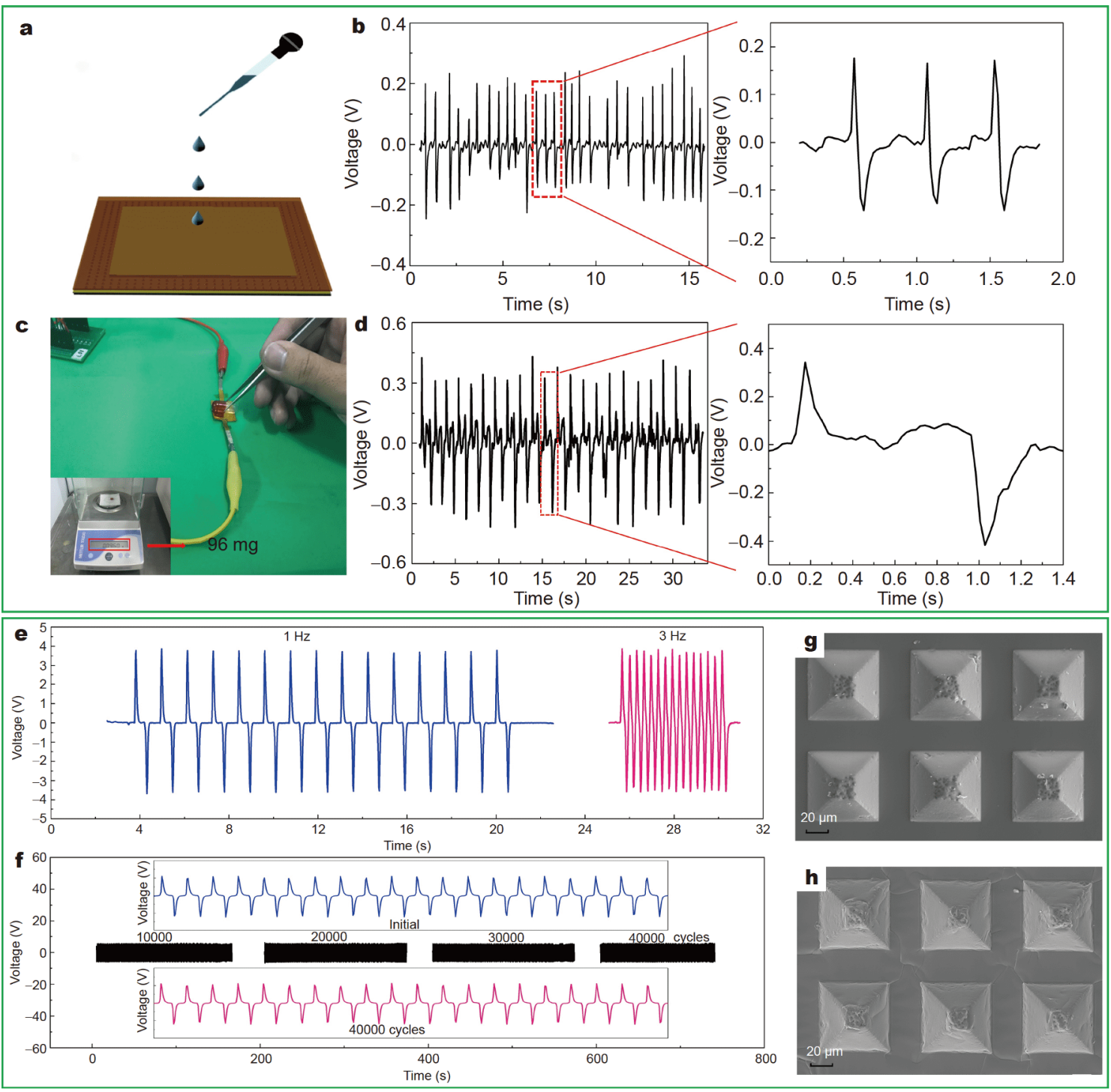

Figure 5 Electronic performance of the TENG tactile sensor induced by (a, b) drops of water ( $\sim 4$ Pa in pressure, $35 \mathrm{mg}$ ) and (c, d) empty capsule ( 96 $\mathrm{mg}$ ) as the loading and unloading process. (e) Measurement of the frequency characteristics of the TENG tactile sensor using a linear motor to apply stable force with different frequencies ( 1 and $3 \mathrm{HZ}$, respectively). (f) The stability test for pressure sensing of the TENG pressure sensor continuously loads and unloads for 40,000 cycles. The open-circuit voltage was measured after every 10,000 cycles. The insets are the enlarged graph at the beginning and 40,000 cycles data. $(\mathrm{g}, \mathrm{h})$ The SEM images of mf-Cu film before and after cyclic test, respectively. 

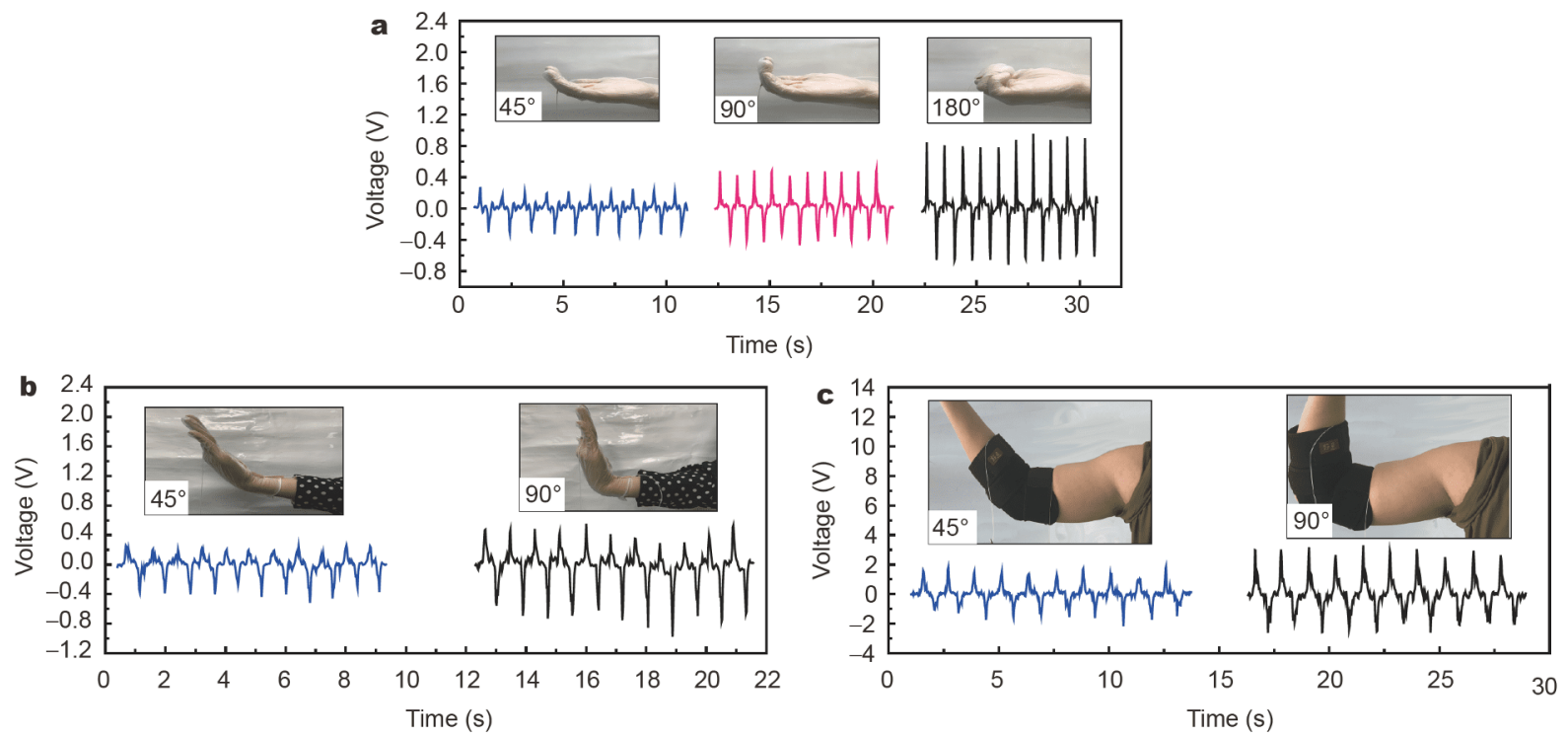

Figure 6 Application of the flexible TENG pressure sensor as an active sensor on the human body. (a) TENG placed on the finger and the opencircuit output voltage measured at different bending angles $45^{\circ} / 90^{\circ} / 180^{\circ}$. (b) TENG integrated with a wrist and the open-circuit output voltage measured at different bending angles $45^{\circ} / 90^{\circ}$. (c) TENG integrated with an elbow and the open-circuit output voltage measured at different bending angles $45^{\circ} / 90^{\circ}$.

of TENG is $60 \mathrm{~ms}$ at $1 \mathrm{~Hz}$. By virtue of the fast response time, the frequency response test was measured showing no obviously decrease from 1 to $3 \mathrm{~Hz}$ (Fig. 5e).

As a flexible wearable sensor, the TENG self-powered sensor needs to release stable signals under a variety of prolonged and repeated tactile stimuli. The stability of the developed sensor was tested under repeated load/unload of a constant force, and the data was recorded after every 10,000 cycles. Fig. $5 f$ shows that the sensor has excellent stability even after 40,000 cycles, and the inset shows the enlarged graphs of the initial state and after 40,000 cycles. Fig. $5 \mathrm{~g}$, h present the SEM images of $\mathrm{mf}-\mathrm{Cu}$ film before and after the cycle test, indicating that the surface microstructure has no significant change. These results reveal that the flexible TENG tactile sensor exhibits great repeatability, stability, durability and fast response. In summary, the high sensitivity and stability of the tactile sensor can be attributed to the PDMS films with uniform micro-frustum-arrays structure, electrospun P(VDFTrFE) nanofibers and a spacer layer with suitable thickness between the two friction layers.

Considering the excellent electrical and mechanical features of the TENG-based sensor, it can be attached to the joints of human body to monitor human body movements for gesture detection [34]. When the joint is bent, the motion causes the relative pressure between the two triboelectric layers and generates the electrical signal output. As shown in Fig. 6a, the flexible TENG was at- tached to latex gloves and bent at different holding angles. With $45^{\circ} / 90^{\circ} / 180^{\circ}$ of finger bending angle, the output voltage is about $0.2 / 0.5 / 0.9 \mathrm{~V}$ (Movie S2 of $180^{\circ}$ ). The possibility of the TENG to detect and distinguish between different movements of the wrist and elbow was also investigated. As shown in Fig. 6b, the flexible TENG sensor was attached to the latex glove on the wrist to measure the output voltage at various wrist bending angles. With $45^{\circ} / 90^{\circ}$ bending angles of wrist, the voltage is approximately $0.2 / 0.5 \mathrm{~V}$ (Movies S3 of $90^{\circ}$ ). Likewise, Fig. 6c shows the output performance of the elbow flexion angles measured by the TENG incorporated in the elbow brackets. With an elbow bending angle of $45^{\circ} / 90^{\circ}$, the output voltage is approximately $1.7 / 3.4 \mathrm{~V}$ (Movies S4 of $90^{\circ}$ ). The TENG can clearly distinguish the different bending angles of different joints and shows great potential in body posture detection. Consequently, our flexible TENG tactile sensor can be used as real-time selfpowered sensor in the field of gesture detection, patient rehabilitation and human-machine interfaces.

\section{CONCLUSIONS}

In this study, a TENG-based flexible tactile sensor composed of $\mathrm{mf}-\mathrm{PDMS} / \mathrm{Cu}$ films, $\mathrm{P}(\mathrm{VDF}-\mathrm{TrFE})$ nanofibers and a spacer has been successfully fabricated with excellent flexibility, high-sensitivity and high stability. The $50 \mu \mathrm{m}$ spacer layer was placed on the middle of two friction layers to ensure the sensor working in contact- 
separation mode. These unique physical structure properties lead to an excellent electrical performance of high sensitivity (2.97 $\mathrm{V} \mathrm{kPa}^{-1}$ ), good stability (over 40,000 cycles), fast response $(60 \mathrm{~ms}$ at $1 \mathrm{~Hz})$, low detection pressure (a water drop $\sim 4 \mathrm{~Pa}, 35 \mathrm{mg}$ ) and good linearity (0.99231 in low pressure region). The high sensitivity and good linearity are due to the micro-frustum-structure and $\mathrm{P}(\mathrm{VDF}-\mathrm{TrFE})$ nanofibers, respectively. Furthermore, the self-powered flexible tactile sensor could be easily attached on various wearable clothes or human skin due to the PDMS as main body and detect different types of the body motion, promising the huge applications in personal healthcare and human-machine interfaces.

\section{Received 28 April 2019; accepted 27 May 2019;} published online 20 June 2019

1 Tee BCK, Chortos A, Berndt A, et al. A skin-inspired organic digital mechanoreceptor. Science, 2015, 350: 313-316

2 Kim DH, Lu N, Ghaffari R, et al. Materials for multifunctional balloon catheters with capabilities in cardiac electrophysiological mapping and ablation therapy. Nat Mater, 2011, 10: 316-323

3 Son D, Lee J, Qiao S, et al. Multifunctional wearable devices for diagnosis and therapy of movement disorders. Nat Nanotech, 2014, 9: 397-404

4 Viventi J, Kim DH, Vigeland L, et al. Flexible, foldable, actively multiplexed, high-density electrode array for mapping brain activity in vivo. Nat Neurosci, 2011, 14: 1599-1605

5 Webb RC, Bonifas AP, Behnaz A, et al. Ultrathin conformal devices for precise and continuous thermal characterization of human skin. Nat Mater, 2013, 12: 938-944

6 Chen S, Lou Z, Chen D, et al. Highly flexible strain sensor based on $\mathrm{ZnO}$ nanowires and $\mathrm{P}(\mathrm{VDF}-\mathrm{TrFE})$ fibers for wearable electronic device. Sci China Mater, 2016, 59: 173-181

7 Deng W, Yang T, Jin L, et al. Cowpea-structured PVDF/ZnO nanofibers based flexible self-powered piezoelectric bending motion sensor towards remote control of gestures. Nano Energy, 2019, 55: 516-525

8 Wang C, Hwang D, Yu Z, et al. User-interactive electronic skin for instantaneous pressure visualization. Nat Mater, 2013, 12: 899-904

9 Shi R, Lou Z, Chen S, et al. Flexible and transparent capacitive pressure sensor with patterned microstructured composite rubber dielectric for wearable touch keyboard application. Sci China Mater, 2018, 61: 1587-1595

10 Zhang Z, Yang J, He X, et al. Plasmonic refractive index sensor with high figure of merit based on concentric-rings resonator. Sensors, 2018, 18: 116

11 Maeda Y, Terao K, Shimokawa F, et al. Reduction of contact force dependence on the mems hardness sensor using reference plane to detect human body hardness. 2015 IEEE Sensors, 2015, 15717259

12 Cao Y, Li T, Gu Y, et al. Fingerprint-inspired flexible tactile sensor for accurately discerning surface texture. Small, 2018, 14: 1703902

13 Lee BY, Kim SU, Kang S, et al. Transparent and flexible high power triboelectric nanogenerator with metallic nanowire-embedded tribonegative conducting polymer. Nano Energy, 2018, 53: 152-159

14 Song Z, Li W, Bao Y, et al. Breathable and skin-mountable strain sensor with tunable stretchability, sensitivity, and linearity via surface strain delocalization for versatile skin activities' recognition. ACS Appl Mater Interfaces, 2018, 10: 42826-42836

15 Choi MK, Park OK, Choi C, et al. Cephalopod-inspired miniaturized suction cups for smart medical skin. Adv Healthcare Mater, 2016, 5: 80-87

16 Chen X, Parida K, Wang J, et al. A stretchable and transparent nanocomposite nanogenerator for self-powered physiological monitoring. ACS Appl Mater Interfaces, 2017, 9: 42200-42209

17 Jian M, Xia K, Wang Q, et al. Flexible and highly sensitive pressure sensors based on bionic hierarchical structures. Adv Funct Mater, 2017, 27: 1606066

18 Wang X, Gu Y, Xiong Z, et al. Silk-molded flexible, ultrasensitive, and highly stable electronic skin for monitoring human physiological signals. Adv Mater, 2014, 26: 1336-1342

19 Choong CL, Shim MB, Lee BS, et al. Highly stretchable resistive pressure sensors using a conductive elastomeric composite on a micropyramid array. Adv Mater, 2014, 26: 3451-3458

20 Lin L, Xie Y, Wang S, et al. Triboelectric active sensor array for self-powered static and dynamic pressure detection and tactile imaging. ACS Nano, 2013, 7: 8266-8274

21 Chen Z, Wang Z, Li X, et al. Flexible piezoelectric-induced pressure sensors for static measurements based on nanowires/graphene heterostructures. ACS Nano, 2017, 11: 4507-4513

22 Park DY, Joe DJ, Kim DH, et al. Self-powered real-time arterial pulse monitoring using ultrathin epidermal piezoelectric sensors. Adv Mater, 2017, 29: 1702308

23 Chen SW, Cao X, Wang N, et al. An ultrathin flexible singleelectrode triboelectric-nanogenerator for mechanical energy harvesting and instantaneous force sensing. Adv Energy Mater, 2017, 7: 1601255

24 Ouyang H, Tian J, Sun G, et al. Self-powered pulse sensor for antidiastole of cardiovascular disease. Adv Mater, 2017, 29: 1703456

25 Jung JH, Lee M, Hong JI, et al. Lead-free $\mathrm{NaNbO}_{3}$ nanowires for a high output piezoelectric nanogenerator. ACS Nano, 2011, 5: 10041-10046

26 Wang S, Lin L, Wang ZL. Triboelectric nanogenerators as selfpowered active sensors. Nano Energy, 2015, 11: 436-462

27 Wen Z, Guo H, Zi Y, et al. Harvesting broad frequency band blue energy by a triboelectric-electromagnetic hybrid nanogenerator. ACS Nano, 2016, 10: 6526-6534

28 Guo Y, Zhang XS, Wang Y, et al. All-fiber hybrid piezoelectricenhanced triboelectric nanogenerator for wearable gesture monitoring. Nano Energy, 2018, 48: 152-160

29 Cheng X, Meng B, Chen X, et al. Single-step fluorocarbon plasma treatment-induced wrinkle structure for high-performance triboelectric nanogenerator. Small, 2016, 12: 229-236

30 Niu S, Wang ZL. Theoretical systems of triboelectric nanogenerators. Nano Energy, 2015, 14: 161-192

31 Fan FR, Lin L, Zhu G, et al. Transparent triboelectric nanogenerators and self-powered pressure sensors based on micropatterned plastic films. Nano Lett, 2012, 12: 3109-3114

32 Li Z, Wang ZL. Air/liquid-pressure and heartbeat-driven flexible fiber nanogenerators as a micro/nano-power source or diagnostic sensor. Adv Mater, 2011, 23: 84-89

33 Wang ZL. Self-powered nanosensors and nanosystems. Adv Mater, 2012, 24: 280-285

34 Lim S, Son D, Kim J, et al. Transparent and stretchable interactive human machine interface based on patterned graphene heterostructures. Adv Funct Mater, 2015, 25: 375-383 
Acknowledgements This work was financially supported by the National Natural Science Foundation of China (51605449, 51675493 and 51705476), the National Key R\&D Program of China (2018YFF0300605), Shanxi "1331 Project" Key Subject Construction (1331KSC), the Applied Fundamental Research Program of Shanxi Province (201601D021070) and Zhangiiakou Science and Technology Research and Development Plan of Zhangjiakou City (1811009B-10).

Author contributions $\quad \mathrm{Yu} \mathrm{J}$ and Hou $\mathrm{X}$ designed the devices and experiments; Yu J, Hou X, Cui M and He J performed the experiments; Yu J, Shi S, Sun Y and Wang C analyzed the data; Yu J wrote the paper with support from Hou X and Chou X. All authors contributed to the general discussion. Yu J and Hou X contributed equally to this work.

Conflict of interest The authors declare no conflict of interest.

Supplementary information Supporting data are available in the online version of the paper.

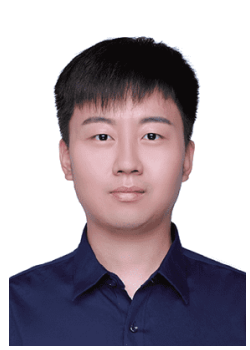

Junbin Yu obtained his BSc degree in 2015 from Tianjin University of Technology and Education. Currently he is a PhD student at the Science and Technology on Electronic Test and Measurement Laboratory in the North University of China (NUC). His main research topic is energy harvesting and flexible self-powered sensor system.

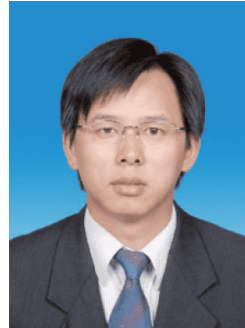

Xiujian Chou works at the School of Instrument and Electronics of the NUC. He received his $\mathrm{PhD}$ degree majored in material physics and chemistry at Tongji University in 2008. Currently he is engaged in intelligent micro/nano device and micro system.

\section{柔性PDMS薄膜摩擦纳米发电机用于监测瞬时力 传感和人体关键运动 \\ 余俊斌 ${ }^{\dagger}{ }^{*}$, 侯晓娟 ${ }^{1 \dagger}$, 崔敏 ${ }^{1}$, 石树正 ${ }^{1,2}$, 何剑 ${ }^{1}$, 孙雅薇 ${ }^{3}$, 王超 ${ }^{1}$, 丑修建 ${ }^{1}$}

摘要 具有出色电学响应和自供电能力的柔性可穿戴传感器已成 为个人医疗保健和人机交互领域重要的研究方向. 本文制备了一 种基于摩擦纳米发电机的柔性自供电触觉传感器, 由具有微四棱 锥台结构的PDMS薄膜、铜电极以及 $\mathrm{P}(\mathrm{VDF}-\mathrm{TrFE})$ 纳米纤维组成. 在外部机械力刺激下, 该传感器能通过接触-分离模式直接产生电 学信号. MEMS工艺制备的均匀可控的微四棱雉台结构和静电纺丝 制备的 P (VDF-TrFE) 纳米纤维, 使得传感器展现出高灵敏度 $\left(2.97 \mathrm{~V} \mathrm{kPa}^{-1}\right.$ )、高稳定性(超过 40000 次循环没有衰减)、快速响 应 (在 $1 \mathrm{~Hz}$ 频率下 $60 \mathrm{~ms}$ )、低压力探测能力(重约 $35 \mathrm{mg}$, 压强约为 $4 \mathrm{~Pa}$ 的水滴) 以及良好的线性度(在低压力区域为 0.99231 ). PDMS薄 膜的超柔韧性和优异的生物相容性, 使得传感器能舒适地贴合在 人体皮肤上. 此外, 所设计的传感器可以通过判断对应的电学响应 信号识别各种人体运动类型. 基于上述优势, 该摩擦纳米发电机有 望应用于姿态监测、健康评估以及人机交互等领域. 